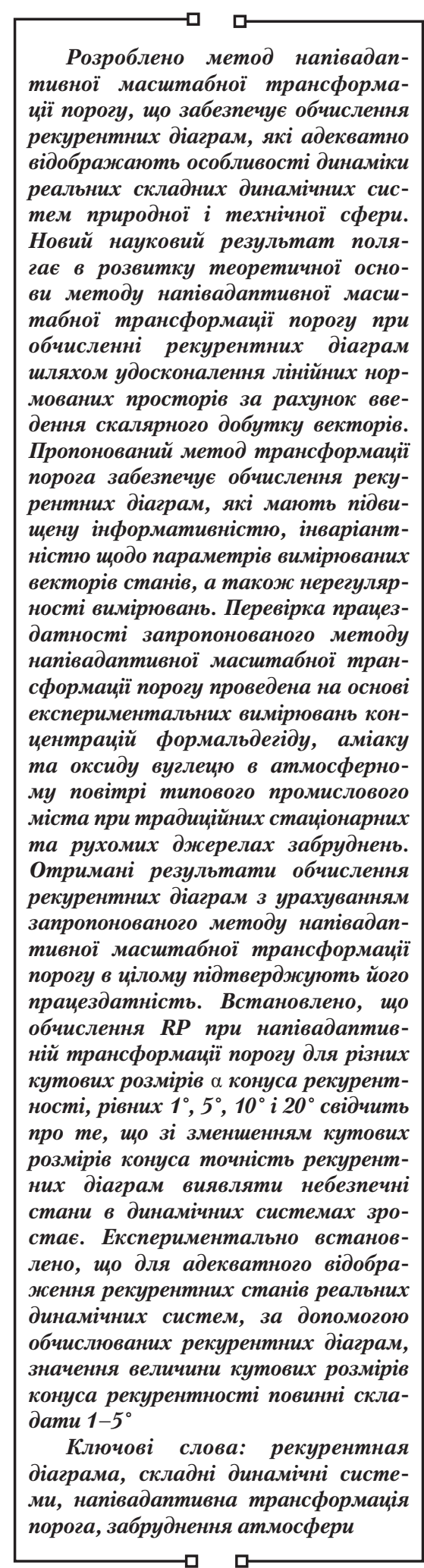

Received date 18.06.2019

Accepted date 30.07.2019

Published date 30.08.2019
UDC 004.932

DOI: $10.15587 / 1729-4061.2019 .176579$

\section{CONSTRUCTION OF THE METHOD} FOR SEMI-ADAPTIVE THRESHOLD SCALING TRANSFORMATION WHEN COMPUTING RECURRENT PLOTS

B. Pospelov

Doctor of Technical Sciences, Professor* E-mail: pospelov@nuczu.edu.ua

E. R y b k a

Doctor of Technical Sciences, Senior Researcher* E-mail: rybka@nuczu.edu.ua

V. Togobyt sk a $\mathrm{PhD*}$

E-mail: leta-yczy@ukr.net

R. Mele s h c he n ko

$\mathrm{PhD}$, Associate Professor Department of Fire and Rescue Training**

E-mail: mel@nuczu.edu.ua

Y u. Danchenko

$\mathrm{PhD}$, Associate Professor

Department of General Chemistry Kharkiv National University of Civil Engineering and Architecture Sumska str., 40, Kharkiv, Ukraine, 61002

E-mail: u_danchenko@ukr.net

T. B u t e n k o

$\mathrm{PhD}$, Senior Research

Department of Organization and Coordination of Research Activities Scientific-Methodical Center of Educational Institutions in the Sphere of Civil Defence Chernyshevska str., 94, Kharkiv, Ukraine, 61023

E-mail: tfire@ukr.net

I. Volkov

Scientific Research Center of Service and Military Activities $* * *$

E-mail: volkov_1972@ukr.net

O. Hafurov

$\mathrm{PhD}$

Department of Technical and Logistics Support*** E-mail: gov20061961@ukr.net

V. Yevsieiev $\mathrm{PhD}$

Department of Special Tactics Preparation $* * *$

E-mail: ua117@ukr.net

*Research Center**

**National University of Civil Defence of Ukraine

Chernyshevska str., 94, Kharkiv, Ukraine, 61023 $* * *$ National Academy of the National Guard of Ukraine

Zakhysnykiv Ukrainy sq., 3, Kharkiv, Ukraine, 61001

Copyright (C 2019, B. Pospelov, E. Rybka, V. Togobytska, R. Meleshchenko,

Yu. Danchenko, T. Butenko, I. Volkov, O. Hafurov, V. Yevsieiev. This is an open access article under the CC BY license (http://creativecommons.org/licenses/by/4.0)

\section{Introduction}

Most of real natural, technical, and social systems exhibit recurrent behavior, which means that certain states of sys- tems repeat over time often [1]. There are methods of recurrent plots (RP) [2] to visualize recurrent behavior of systems. They represent the basis for methods of quantitative analysis of recurrence (RQA). Recently, scientists use RP and RQA 
methods in various sciences [1,3-6]. A paper [7] presents the current state of RP and RQA methods. Capabilities of RQA methods depend on adequacy of RP mapping of the recurrent behavior of real systems substantially. Therefore, one should pay special attention to accuracy of mapping of recurrent states of systems in RP for real systems. Accuracy of mapping of recurrent states of systems in RP calculation depends on specific measurement conditions and methods of processing of measurement information implemented in the case. One of the limitations of the known methods of RP calculation for a given norm functional [7] is the dependence of accuracy of mapping of recurrent states on the threshold used, that is the threshold uncertainty of methods of RP calculation. Under various and changing conditions, which are characteristic for most of applications, the threshold uncertainty of methods reduces accuracy in displaying the recurrent states of real systems. An existing variety of types of real systems, conditions for measurement of their states, and presence of threshold uncertainty of known methods for RP calculation, which reduce accuracy of displaying of recurrent states. Therefore, the necessity to increase accuracy of mapping of RP under real conditions creates the problem of improvement of known methods for calculation of RP.

\section{Literature review and problem statement}

RP and RQA methods are applicable to results of real measurements of a state of dynamic systems. Therefore, they are an attractive tool for studies on behavior of complex dynamic systems in various fields [2]. The state vector of dynamical systems has a finite size usually, and it varies in time. In time-discrete measurements, the state vector determines coordinates of a point, which moves along a certain trajectory of system states, in the corresponding phase space at a fixed moment in time. The recurrence of system states at different moments of time means that arbitrary points of the indicated trajectory turn out to be close in some sense [4]. RP methods, in contrast to the methods of correlation dimension [8,9], give a possibility to map multidimensional trajectories of states of complex dynamic systems in a 2-dimensional phase space. The methods take into account perturbations and distortions in measurement channels in the structure of dynamics of system states. Following paper [10], it is possible to judge the structure of dynamics of states of a whole system by measurement of only one measured coordinate of the state vector. However, such measurement creates additional uncertainty in RP calculation in the form of a parameter of the embedding dimension. Work [11] considers a method for forecasting of dangerous states of atmospheric pollution in industrial cities based on a measure of recurrence of states for one coordinate only. There are RP calculation and the proposed measures of recurrence performed for a usual metric (distance) in the space of all real numbers. The work does not consider other types of metrics and their corresponding metric spaces. Paper [12] considers a method for RP calculation for the state vector of wind velocity in five regions of Nigeria. The study considers a linear space with Euclidean metric only. It does not consider other metrics and spaces, and large-scale transformation of thresholds in RP calculation. There is parameterization of RP calculation under conditions of artifacts in the reconstructed state vector [10] considered in work [13]. The study considers Euclidean metric of space. It does not consider other types of metrics and threshold scaling. Paper [14] studies methods of
RP calculation for the reconstructed vector of states of the Earth's magnetosphere. The paper studies normalized linear spaces with maximum metrics and Chebyshev metrics only. Work [15] considers a solution for the problem of recognition and classification of various human motor activities. It proposes to exclude the threshold and to calculate distance matrices instead of RP to overcome the threshold uncertainty of methods of RP calculation under such conditions associated with difficulties and errors. However, such approach is not constructive in our opinion, since it leads to an additional problem of determination of recurrent states by the distance matrix. The solution for this problem is possible by the use of a neural network, which has its known disadvantages and limitations. Paper [16] considers the use of the method of RP calculation in a space with a Euclidean metric to analyze behavior of various biological systems. Authors note that measurements, time delays, dimension of an embedding and a threshold influence accuracy of RP calculation affect accuracy of RP calculation. Authors specify that the threshold should be different for each set of measurements, but it should not exceed $10 \%$ of the maximum diameter, which corresponds to the phase space.

A method of RP calculation for irregular measurements based on a metric space with a metric in the form of a distance between the corresponding measurements is important for applications [17]. Work [7] presents general recommendations to overcome threshold uncertainty. Authors note that it is possible to overcome threshold uncertainty by fixing a threshold value based on requirements of a specific research task. Authors of paper [18] state that a threshold should be a function of the standard deviation in measurements in order to identify characteristics of dynamics of states of a real system during RP calculation. However, they do not identify a type of the function itself. They only note that such method of determination of a threshold can be useful for any method of RP calculation. Authors of work [19] consider combination of a multilevel network approach and recurrent networks for analysis of behavior of multidimensional states. However, the work considers the state vector in a normed space with a Euclidean metric only. It does not consider other types of metrics and spaces. Paper [20] describes new methods of RP calculation for drilling data with the purpose to study dynamics of an ore body formation system. The study considers a normed space of a general form with an arbitrary metric without its specification. Work [1] studies a method of RP calculation for the state vector of dynamic systems in an abstract phase space with a trivial metric. Paper [21] studies features of RP calculation for localization of transient processes in systems [21]. It notes that it is possible to use spaces of states with different metrics in the methods of RP calculation. But it does not consider an impact of metrics and threshold scaling on the result of RP calculation. Authors of work [22] consider application of the RP method of carbon monoxide concentration for early detection of indoor fires [22]. However, studies consider measurements in one-dimensional space with ordinary and exponential distance metrics only. And studies do not consider multidimensional spaces and threshold scaling. Work [23] considers methods and devices for self-adjusting detection of dangerous factors of early fires in a one-dimensional case. Authors note that the choice of a threshold is a key to reliable detection [24].

Our analysis shows that the base of known methods of $\mathrm{RP}$ calculation are normalized linear spaces of finite dimension and various types of metrics. The methods of RP calcu- 
lation in linear spaces with uniform, Euclidean, and maximal metric that define the corresponding proper topology of spaces, are more studied methods for now. Therefore, RP will be different for the same system calculated in spaces with different metrics. Methods of RP calculation in spaces with other types of metrics are less studied. In addition to the metric, the recurrence threshold affects accuracy of mapping of recurrent states of systems in RP. Therefore, the problem of selection of the recurrence threshold becomes a key problem for the methods of calculation of RP, which map real recurrence of system states adequately [1]. At present, there are several known threshold selection heuristics [7, 25]. However, they are applicable for particular conditions. They are limited to well-known metrics and they have significant implementation disadvantages. So, we can state that an important and unresolved part of the problem of improvement of the well-known methods of RP calculation is a lack of threshold transformation methods, which provide a reflection of recurrence of states adequate to real systems.

\section{The aim and objectives of the study}

The objective of the study is to develop a method of semi-adaptive scaling transformation of the threshold during calculation of recurrent plots. The method should provide an adequate mapping of recurrent states of real systems in natural and technical spheres.

We set the following tasks to achieve the objective:

- theoretical substantiation of the method of semi-adaptive scaling transformation of the threshold during calculation of recurrent plots for complex dynamic systems;

- experimental verification of efficiency of the method of semi-adaptive scaling transformation of the threshold during calculation of recurrent plots using an example of real dynamics of states of dangerous atmospheric pollutants.

\section{Theoretical substantiation of the method of semi-adaptive scaling transformation of the threshold during calculation of recurrent plots}

The base of RP calculation methods are measurements of an arbitrary $m$-dimensional $Z_{i}$ vector of states of the dynamic system under study at $i$ discrete time moments. We can determine them from expression

$$
\begin{gathered}
R_{i, j}^{m,}=\Theta\left(\varepsilon-\left\|Z_{i}-Z_{j}\right\|\right), \quad Z_{i} \in \Omega^{m}, \\
Z_{j} \in \Omega^{m}, \quad i, j=0,1,2, \ldots, N_{S}-1,
\end{gathered}
$$

where $\Theta\left({ }^{*}\right)$ is the Heaviside function; $\varepsilon$ is the recurrence threshold or a limit size of a neighborhood of a point characterized by $Z_{i}$ vector at $i$ time moment in the space under study; $\left\|^{*}\right\|$ is the sign of the norm in the space; $N_{S}$ is the maximum number of measurements of $Z_{i}$ vector of system states. Following expression (1), RP for an arbitrary $Z_{i}$ vector will depend on the chosen norm and $\varepsilon$ recurrence threshold in the general case. For a fixed $\varepsilon$ threshold, different norms will affect a series of recurrent points in (1). If the norm is given, a too small $\varepsilon$ threshold can lead to absence of recurrence points in RP, although they exist in a real system in fact. On the other hand, if $\varepsilon$ threshold is too large, almost every point, which corresponds to the state vector in the phase space, will turn out to be recurrent to any other point. It means that, for a given norm, the choice of $\varepsilon$ recurrence threshold should be consistent and compromise in (1). In the general case, the development of threshold selection methods is necessary in (1) to ensure calculation of RP, which are adequate for dynamic systems under study. The presence of measurement noise distorts RP additionally (1). Therefore, a paper [26] proposes to choose a fixed $\varepsilon$ threshold equal to several percent of the maximum diameter of the phase space under study in (1). However, the threshold should not exceed $10 \%$ of the average or maximum diameter of the phase space. The basis of another approach is a given model of dynamics of a system state in the form of a composition of a deterministic component and a random component with $\sigma$ given standard deviation $\sigma$ [27]. We must choose $\varepsilon$ threshold according to the condition $\varepsilon>5 \sigma$ for an adequate mapping of RP.

Absence of adaptation of the threshold to the specified norm and conditions for measurement of system states, complexity and non-universality of the known norms and metrics of spaces in the methods of RP calculation limit ability to map recurrent states, which are adequate to real systems. Therefore, there is a need to develop a method for adaptive threshold transformation taking into account a given norm for RP calculation. The method provides an adequate mapping of dynamics of recurrent states of complex systems.

We can consider the proposed transition to some improved space due to introduction of an additional geometric characteristic in the form of a scalar product of two vectors as the basis for development of such method [28]. Following [28], the scalar product will generate the norm in the improved space. The norm, in turn, will generate the corresponding metric of the following form

$$
d\left(Z_{\mathrm{i}}, \mathrm{Z}_{\mathrm{j}}\right)=\left\|Z_{\mathrm{i}}-\mathrm{Z}_{\mathrm{j}}\right\|=\sqrt{\left(Z_{\mathrm{i}}-\mathrm{Z}_{\mathrm{j}}\right)^{T}\left(Z_{\mathrm{i}}-\mathrm{Z}_{\mathrm{j}}\right)} .
$$

Taking into account (2), we can describe the RP calculation method (1) for arbitrary $m$-dimensional $Z_{i}$ state vectors of the system under study in the considered space of the improved structure by the expression

$$
R_{i, j}^{m, \varepsilon}=\Theta\left(\varepsilon-\sqrt{\left(Z_{\mathrm{i}}-\mathrm{Z}_{\mathrm{j}}\right)^{T}\left(Z_{\mathrm{i}}-\mathrm{Z}_{\mathrm{j}}\right)}\right) .
$$

We can see from expression (3) that the norm in RP calculation is equivalent to the norm of the method (1) generating the Euclidean metric in the space of an improved structure. An important feature of method (3) is that the scalar product of the difference of the corresponding state vectors of a system replaces calculation of the Euclidean metric. $\varepsilon$ threshold is fixed in the method (3). We can select it based on well-known approaches and recommendations [26,27]. In contrast to (1), the scalar product $\left(Z_{i}-Z_{j}\right)^{\mathrm{T}}\left(Z_{i}-Z_{j}\right)$ in (3) contains information not only on the squared distance between $Z_{i}$ and $Z_{j}$ state vectors of the system with the Euclidean metric, but also information on the squared lengths of the mentioned vectors and the mutual angular position of the vectors in the considered improved space. The information contained in the scalar product $\left(Z_{i}-Z_{j}\right)^{\mathrm{T}}\left(Z_{i}-Z_{j}\right)$ in (3) makes possible to develop and to implement the method of semi-adaptive scaling transformation of $\varepsilon$ threshold for the Euclidean metric in (1). The essence of the method is that $\varepsilon$ threshold is not fixed. We determine it by adaptive scaling transformation in accordance with the measured $Z_{i}$ 
and $Z_{j}$ state vectors for a fixed value of $\gamma$ angle between the mentioned vectors in the space under study. Following the cosine theorem, we can show that the following expression determines the semi-adaptive threshold

$$
\varepsilon\left(Z_{i}, Z_{j}, \gamma\right)=\sqrt{Z_{i}^{\mathrm{T}} Z_{i}+Z_{j}^{\mathrm{T}} Z_{j}-2 \sqrt{Z_{i}^{\mathrm{T}} Z} \sqrt{Z_{j}^{\mathrm{T}} Z_{j}} \cos \gamma}
$$

Based on the expression (4), $\varepsilon\left(Z_{i}, Z_{j}, \gamma\right)$ threshold is adaptive to $Z_{i}$ and $Z_{j}$ state vectors and depends on $\gamma$ angle between the vectors. The value of $\gamma$ angle sets the boundary level of the angular recurrence of states of the system. If we set $\gamma=\pi \alpha / 180$ in (4), then $\alpha$ value specifies the acceptable angular sector in degrees. We consider $Z_{j}$ state vectors as recurrent to $Z_{i}$ state vectors at $i$ time moment in the improved space within the mentioned angular sector. $\alpha$ value of the acceptable angular sector determines the recurrence for nonzero state vectors. Therefore, the calculation of (3) at the threshold (4) ensures the invariance of RP to measurements of state vectors. The RP calculation method (3) corresponds to the mapping of dynamics in the case of conical neighborhoods of recurrence instead of traditional spherical neighborhoods.

5. Experimental verification of operability of the method of semi-adaptive scaling threshold transformation

We tested operability of the proposed method based on experimental data on measurements of real dynamics of typical dangerous gas pollutants in the urban atmosphere. We know that the main sources of urban air pollution are motor vehicles [29], fires [30, 31] and accidents at critical infrastructure facilities [32]. There is a stable relationship between global atmospheric pollution and the greenhouse effect, acid rain [33], and pollution of aquiferous layers [34]. Therefore, we chose formaldehyde $\left(\mathrm{CH}_{2} \mathrm{O}\right)$, ammonia $\left(\mathrm{NH}_{3}\right)$, and carbon monoxide (CO) as the measured components of the state vector of atmospheric pollution. Paper [35] describes experimental research methodology in detail. We measured the concentration of atmospheric gas pollution with a portable DRÄGER PAC 7000 gas analyzer (Germany). It provided a short response time (up to $20 \mathrm{~s})$ and high measurement accuracy $\left(0.01 \mathrm{mg} / \mathrm{m}^{3}\right)$. We chose the interval of measurements of exceeding of the maximum permissible concentrations (MPC) of the mentioned pollutants in the atmosphere as the test interval. It was from 13:00 on May 3, $2018(i=490)$ to 01:00 on May 11, $2018(i=520)$. Fig. 1 shows the results of measurements of dynamics of the concentrations of formaldehyde (red curve), ammonia (blue curve) and carbon monoxide (brown curve) in the atmosphere relatively to their MPC in the test interval.
One can see in Fig. 1 that the interval between 504 and 511 points is the most dangerous state of atmospheric pollution in the test interval. We calculated RP in accordance with (1) for $\varepsilon$ fixed threshold equal to 4 arbitrary units for normed spaces with a uniform, Euclidean, and maximal metric firstly to verify effectiveness of the proposed method according to the data in Fig. 1. Fig. 2 shows the corresponding diagrams calculated for this case. Fig. 3 illustrates the effect of the threshold value on RP structure is with a lower threshold $\varepsilon=2$.
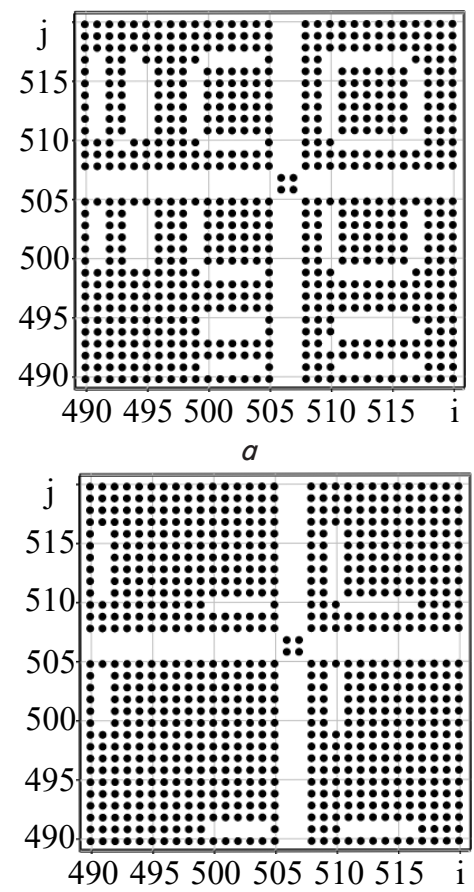

$b$

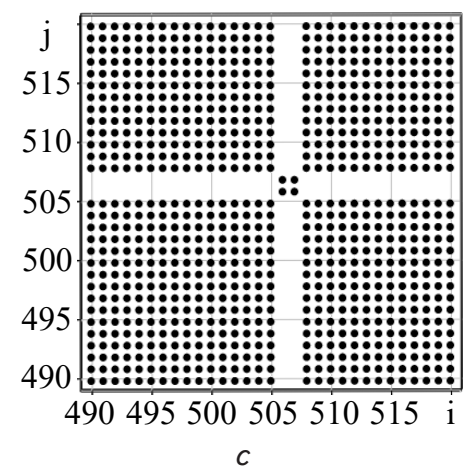

Fig. 2. RP of atmospheric pollution in the test interval for the threshold $\varepsilon=4$ and various metrics: $a-$ uniform metric; $b$ - Euclidean metric; $c$ - maximum metric

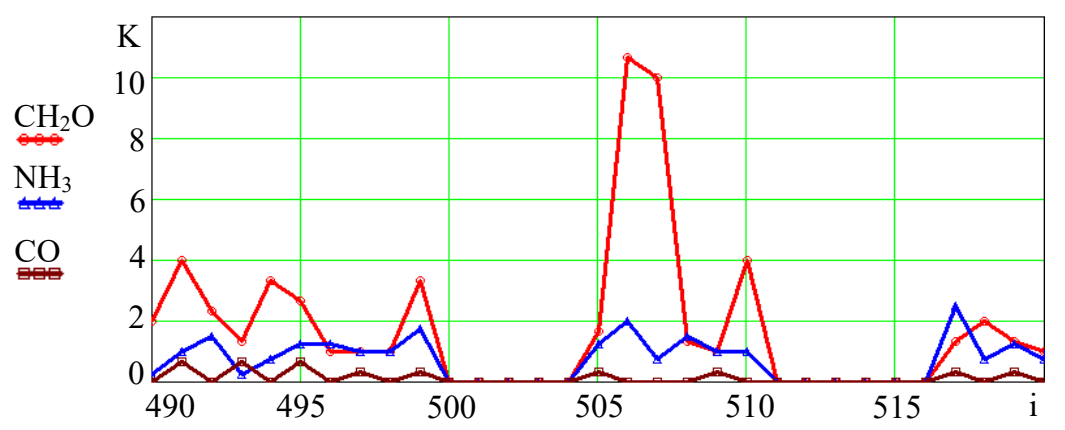

Fig. 1. The dynamics of concentrations of pollutants relative to MPC in the atmosphere
Then, during verification, we calculated $\mathrm{RP}$ for the same fixed $\varepsilon$ threshold values equal to 4 and 2 arbitrary units in the proposed improved space, following the expression (4). Fig. 4 shows the results of RP calculations of for this case.

Next, we calculated RP of atmospheric pollution in the test interval, taking into account the implementation of the proposed method (3), but for various $\alpha$ values of the acceptable angle of recurrence cone when implementing the method of semi-adaptive transformation of the threshold (4). Fig. 5 
shows the results of the calculations for $\alpha$ values of $1^{\circ}, 5^{\circ}$, $10^{\circ}$, and $20^{\circ}$.
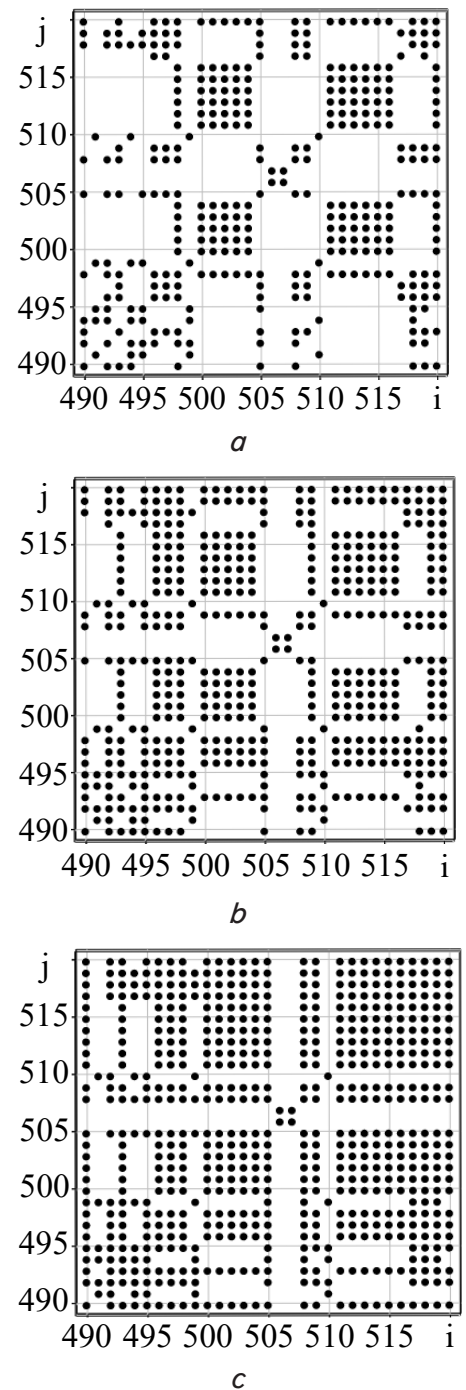

Fig. 3. RP of atmospheric pollution in the test interval for the threshold $\varepsilon=2$ and various metrics: $a-$ uniform metric; $b$ - Euclidean metric; $c$ - maximum metric

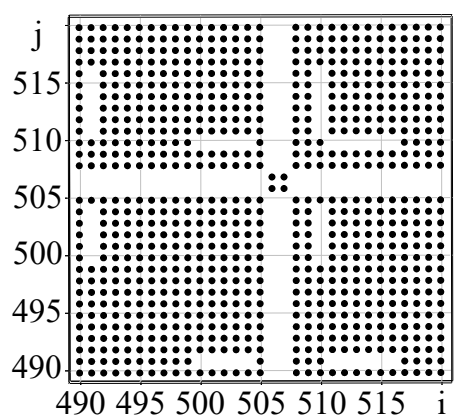

490495500505510515

a

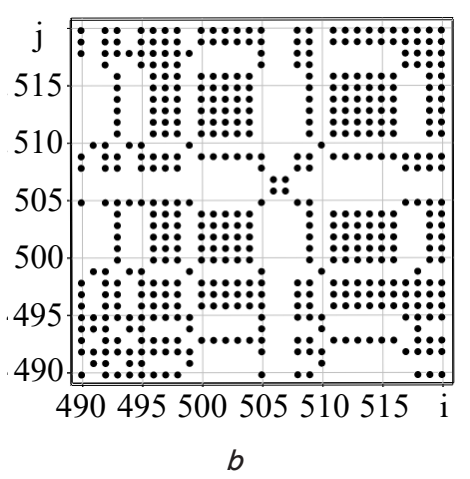

$b$
Fig. 4. RP of air pollution it the test interval in the improved space for the threshold values: $a-\varepsilon=4 ; b-\varepsilon=2$

The red oval denotes the region of recurrence on RP in Fig. 5. It maps presence of a laminar state in the atmosphere. Such state has dangerous excesses of MPC for formaldehyde and ammonia and corresponds to 507 points of experimental data.
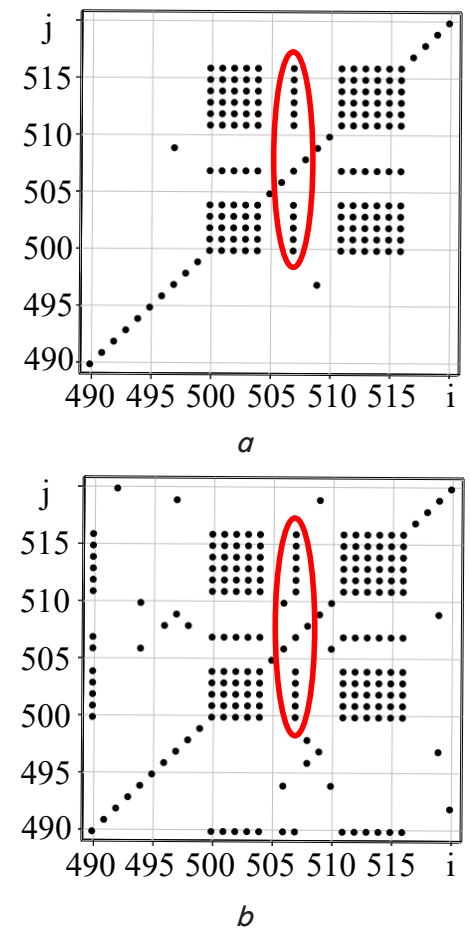

Fig. 5. RP of atmospheric pollution in the test interval during the implementation of semi-adaptive transformation of the threshold for various values of the acceptable angle of the recurrence cone: $a-\alpha=1^{\circ}$; $b-\alpha=5^{\circ} ; c-\alpha=10^{\circ} ; d-\alpha=20^{\circ}$

6. Discussion of results from experimental verification of the proposed method

The results of measurement (Fig. 1) of the dynamics of the excess of MPC for concentrations of formaldehyde (red curve), ammonia (blue curve) and carbon monoxide (brown curve) in the atmosphere indicate an unequal level of pollution in the test interval under study. The most dangerous state of atmospheric pollution is the interval between 504 and 511 measurement points (10-fold excess of MPC for formaldehyde and a double excess for ammonia).

The experimental verification of the well-known method of RP calculation for a fixed threshold and various metrics of the normalized space used indicates the heterogeneity of the structural mappings shown in Fig. 2, 3. The form of the mapped RP structure depends on the threshold and space metric. Following the structures in Fig. 2, 3, for a fixed threshold $\varepsilon$, we can see that RP make possible to interpret recurrent states of systems in the sense of proximity of distances only in the case of using the Euclidean metric of spaces. Vertical and horizontal sets of black dots on $\mathrm{RP}$ are characteristic for laminar states of a polluted atmosphere, when concentrations of pollution are not dispersed by the atmosphere by various reasons. Such conditions are dangerous. They can cause many emergency situations of delayed manifestation. We should note that laminar states have different degrees of accuracy for all considered metrics. The set of white dots on the RP characterizes absence of recurrence of the corresponding states of the polluted atmosphere. White points may appear due to a possible short-term loss of recurrence of states with 
subsequent transition to a laminar state or lack of regularity of measurements. In the general case, a decrease in the fixed $\varepsilon$ threshold leads to a greater detailing of RP structure (alternation of black and white dots) for all considered metrics.

$\mathrm{RP}$ of the vector of states of atmospheric pollution in the test interval (Fig. 1) in the improved space for various values of the fixed $\varepsilon$ threshold (Fig. 4) indicate the coincidence of RP structures for the same threshold values for the Euclidean metric. Because the metric used in (3) is equivalent to the Euclidean metric in determination of the norm in (1). The definition of the norm in (3) is slightly simpler in comparison with (1) in terms of calculations. An important feature of the representation of the norm in (3) in an improved space compared to (1) is the possibility of implementation of the proposed method of semi-adaptive scaling threshold transformation, which is absent in case (1).

Analysis of RP in Fig. 5 and areas marked with an oval of red color shows that it is possible to achieve greater information content and adequacy of mapping according to real systems when implementing the proposed method in the improved space. We compared the results of experimental studies with the theoretical basis of the proposed method and revealed that the method has the important feature to map dangerous states in dynamical systems characterized by their laminar states. And there is no need in information on the meteorological situation in case of atmospheric pollution. We established that the value of $\alpha$ affects accuracy of mapping of recurrent states, and ability of RP to detect dangerous states in various dynamic systems in the case of implementation of the method of semi-adaptive transformation of the threshold. So, with a decrease in $\alpha$, the accuracy of mapping of RP of the real states of dynamic systems increases. However, we must take into account that recurrent states will not be mapped in RP at $\alpha=0$. We established experimentally that it is necessary to choose working values of $\alpha$ from the interval: $1-5^{\circ}$. Rectangular clusters of black dots in $\mathrm{RP}$ indicate presence of corresponding recurrent states in Fig. 5. However, they are not features of the studied atmospheric pollution, since they are appeared due to features of the primary processing used and correspond to the moments of absence of measurements. In addition, the scalar product of vectors generates three-parameter conical neighborhoods of recurrence instead of the traditional one-parameter spherical neighborhoods in the improved space for the Euclidean metric. The paradigm of a three-parameter conical neighborhood of state recurrence changes the traditional concept of spherical recurrence to the concept of extended generalized recurrence. Introduction of three-parameter conical neighborhoods made possible to develop a method of semi-adaptive scaling transformation of the threshold. Adaptation of the threshold takes place to the measured state vectors of the system, providing measuring invariance of RP. And the angular size of the state recurrence cone provides the required accuracy of RP calculation. Therefore, the proposed method of semi-adaptive scaling transformation of the threshold provides calculation of $\mathrm{RP}$, which are adequate to real dynamic systems.

We can implement applications based on the developed method for early detection of dangerous pollution of the surface layer of the atmosphere with the purpose of using it in intelligent urban transport control systems for development of decision support systems under conditions of possible preconditions for emergency situations and timely warning of the population.

A possible limitation of the study is validity of the assumption of nonzero values of the measured state vectors of the system under study. There is a false recurrence of states for zero state vectors, which correspond to the case of absence of measurements usually. The false recurrence is undesirable in quantitative analysis. Therefore, elimination of false recurrence can be a possible direction for the development of the study.

\section{Conclusions}

1. We developed a method of semi-adaptive scaling transformation of the threshold. The method provides RP calculation, which maps recurrence of states of real dynamic systems in natural and technical spheres adequately. A new scientific result is the development of the theoretical basis of the method of semi-adaptive scaling transformation of the threshold during RP calculation based on improvement of linear normed spaces. The developed method of semi-adaptive scaling transformation of the threshold provides RP calculation with increased information content, low complexity, invariance with respect to a length and size of state vectors, and possibility of implementation under irregular measurements.

2 . We tested the operability of the proposed method of semi-adaptive scaling transformation of the threshold based on experimental measurements of concentrations of formaldehyde, ammonia, and carbon monoxide in the atmosphere of a typical industrial city with traditional stationary and mobile sources of pollution. Taking into account the proposed method, the obtained results of RP calculation confirmed its operability. We found that RP calculation during the semi-adaptive transformation of the threshold for various $\alpha$ angular sizes of a cone of recurrence equal to $1^{\circ}, 5^{\circ}, 10^{\circ}$, and $20^{\circ}$ indicates that, the accuracy of RP in detection of dangerous states in dynamic systems increases with a decrease in $\alpha$ angular dimension of a cone. We established experimentally that values of $\alpha$ should be $1-5^{\circ}$ for adequate mapping of recurrent states of real dynamic systems with the use of RP calculation.

\section{References}

1. Webber, C. L., Marwan, N. (Eds.) (2015). Recurrence Quantification Analysis. Understanding Complex Systems. doi: https://doi.org/ 10.1007/978-3-319-07155-8

2. Marwan, N., Webber, C. L., Macau, E. E. N., Viana, R. L. (2018). Introduction to focus issue: Recurrence quantification analysis for understanding complex systems. Chaos: An Interdisciplinary Journal of Nonlinear Science, 28 (8), 085601. doi: https://doi.org/ $10.1063 / 1.5050929$ 
3. Souza, E. G., Viana, R. L., Lopes, S. R. (2008). Using recurrences to characterize the hyperchaos-chaos transition. Physical Review E, 78 (6). doi: https://doi.org/10.1103/physreve.78.066206

4. Javorka, M., Trunkvalterova, Z., Tonhajzerova, I., Lazarova, Z., Javorkova, J., Javorka, K. (2008). Recurrences in heart rate dynamics are changed in patients with diabetes mellitus. Clinical Physiology and Functional Imaging, 28 (5), 326-331. doi: https://doi.org/ 10.1111/j.1475-097x.2008.00813.x

5. Oya, S., Aihara, K., Hirata, Y. (2014). Forecasting abrupt changes in foreign exchange markets: method using dynamical network marker. New Journal of Physics, 16 (11), 115015. doi: https://doi.org/10.1088/1367-2630/16/11/115015

6. Carrión, A., Miralles, R., Lara, G. (2014). Measuring predictability in ultrasonic signals: An application to scattering material characterization. Ultrasonics, 54 (7), 1904-1911. doi: https://doi.org/10.1016/j.ultras.2014.05.008

7. Marwan, N. (2011). How to avoid potential pitfalls in recurrence plot based data analysis. International Journal of Bifurcation and Chaos, 21 (04), 1003-1017. doi: https://doi.org/10.1142/s0218127411029008

8. Pospelov, B., Andronov, V., Rybka, E., Meleshchenko, R., Gornostal, S. (2018). Analysis of correlation dimensionality of the state of a gas medium at early ignition of materials. Eastern-European Journal of Enterprise Technologies, 5 (10 (95)), 25-30. doi: https://doi.org/10.15587/1729-4061.2018.142995

9. Andronov, V., Pospelov, B., Rybka, E. (2017). Development of a method to improve the performance speed of maximal fire detectors. Eastern-European Journal of Enterprise Technologies, 2 (9 (86)), 32-37. doi: https://doi.org/10.15587/1729-4061.2017.96694

10. Takens, F. (1981). Detecting strange attractors in turbulence. Dynamical Systems and Turbulence, Warwick 1980, 366-381. doi: https://doi.org/10.1007/bfb0091924

11. Pospelov, B., Andronov, V., Rybka, E., Popov, V., Semkiv, O. (2018). Development of the method of frequencytemporal representation of fluctuations of gaseous medium parameters at fire. Eastern-European Journal of Enterprise Technologies, 2 (10 (92)), 44-49. doi: https://doi.org/10.15587/1729-4061.2018.125926

12. Adeniji, A. E., Olusola, O. I., Njah, A. N. (2018). Comparative study of chaotic features in hourly wind speed using recurrence quantification analysis. AIP Advances, 8 (2), 025102. doi: https://doi.org/10.1063/1.4998674

13. Wendi, D., Marwan, N., Merz, B. (2018). In Search of Determinism-Sensitive Region to Avoid Artefacts in Recurrence Plots. International Journal of Bifurcation and Chaos, 28 (01), 1850007. doi: https://doi.org/10.1142/s0218127418500074

14. Donner, R. V., Balasis, G., Stolbova, V., Georgiou, M., Wiedermann, M., Kurths, J. (2019). Recurrence Based Quantification of Dynamical Complexity in the Earth's Magnetosphere at Geospace Storm Timescales. Journal of Geophysical Research: Space Physics, 124 (1), 90-108. doi: https://doi.org/10.1029/2018ja025318

15. Garcia-Ceja, E., Uddin, M. Z., Torresen, J. (2018). Classification of Recurrence Plots' Distance Matrices with a Convolutional Neural Network for Activity Recognition. Procedia Computer Science, 130, 157-163. doi: https://doi.org/10.1016/j.procs.2018.04.025

16. Neves, F. M., Viana, R. L., Pie, M. R. (2017). Recurrence analysis of ant activity patterns. PLOS ONE, 12 (10), e0185968. doi: https://doi.org/10.1371/journal.pone.0185968

17. Ozken, I., Eroglu, D., Breitenbach, S. F. M., Marwan, N., Tan, L., Tirnakli, U., Kurths, J. (2018). Recurrence plot analysis of irregularly sampled data. Physical Review E, 98 (5). doi: https://doi.org/10.1103/physreve.98.052215

18. Schinkel, S., Dimigen, O., Marwan, N. (2008). Selection of recurrence threshold for signal detection. The European Physical Journal Special Topics, 164 (1), 45-53. doi: https://doi.org/10.1140/epjst/e2008-00833-5

19. Eroglu, D., Marwan, N., Stebich, M., Kurths, J. (2018). Multiplex recurrence networks. Physical Review E, 97 (1). doi: https://doi.org/ 10.1103/physreve.97.012312

20. Oberst, S., Niven, R., Ord, A., Hobbs, B., Lester, D. (2017). Application of recurrence plots to orebody exploration data. Conference: Target. At University Club, University of Western Australia.

21. Webber, C. L., Ioana, C., Marwan, N. (Eds.) (2016). Recurrence Plots and Their Quantifications: Expanding Horizons. Springer Proceedings in Physics. doi: https://doi.org/10.1007/978-3-319-29922-8

22. Pospelov, B., Andronov, V., Rybka, E., Meleshchenko, R., Borodych, P. (2018). Studying the recurrent diagrams of carbon monoxide concentration at early ignitions in premises. Eastern-European Journal of Enterprise Technologies, 3 (9 (93)), 34-40. doi: https://doi.org/10.15587/1729-4061.2018.133127

23. Pospelov, B., Andronov, V., Rybka, E., Skliarov, S. (2017). Design of fire detectors capable of self-adjusting by ignition. Eastern-European Journal of Enterprise Technologies, 4 (9 (88)), 53-59. doi: https://doi.org/10.15587/1729-4061.2017.108448

24. Pospelov, B., Andronov, V., Rybka, E., Skliarov, S. (2017). Research into dynamics of setting the threshold and a probability of ignition detection by selfadjusting fire detectors. Eastern-European Journal of Enterprise Technologies, 5 (9 (89)), 43-48. doi: https://doi.org/10.15587/1729-4061.2017.110092

25. Beim Graben, P., Hutt, A. (2015). Detecting event-related recurrences by symbolic analysis: applications to human language processing. Philosophical Transactions of the Royal Society A: Mathematical, Physical and Engineering Sciences, 373 (2034), 20140089. doi: https://doi.org/10.1098/rsta.2014.0089 
26. Mindlin, G. M., Gilmore, R. (1992). Topological analysis and synthesis of chaotic time series. Physica D: Nonlinear Phenomena, 58 (1-4), 229-242. doi: https://doi.org/10.1016/0167-2789(92)90111-y

27. Thiel, M., Romano, M. C., Kurths, J., Meucci, R., Allaria, E., Arecchi, F. T. (2002). Influence of observational noise on the recurrence quantification analysis. Physica D: Nonlinear Phenomena, 171 (3), 138-152. doi: https://doi.org/10.1016/s0167-2789(02)00586-9

28. Pospelov, B., Andronov, V., Meleshchenko, R., Danchenko, Y., Artemenko, I., Romaniak, M. et. al. (2019). Construction of methods for computing recurrence plots in space with a scalar product. Eastern-European Journal of Enterprise Technologies, 3 (4 (99)), 37-44. doi: https://doi.org/10.15587/1729-4061.2019.169887

29. Kondratenko, O. M., Vambol, S. O., Strokov, O. P., Avramenko, A. M. (2015). Mathematical model of the efficiency of diesel particulate matter filter. Naukovyi Visnyk Natsionalnoho Hirnychoho Universytetu, 6, 55-61.

30. Vasiliev, M. I., Movchan, I. O., Koval, O. M. (2014). Diminishing of ecological risk via optimization of fire-extinguishing system projects in timber-yards. Naukovyi Visnyk Natsionalnoho Hirnychoho Universytetu, 5, 106-113.

31. Dubinin, D., Korytchenko, K., Lisnyak, A., Hrytsyna, I., Trigub, V. (2017). Numerical simulation of the creation of a fire fighting barrier using an explosion of a combustible charge. Eastern-European Journal of Enterprise Technologies, 6 (10 (90)), 11-16. doi: https://doi.org/10.15587/1729-4061.2017.114504

32. Semko, A., Rusanova, O., Kazak, O., Beskrovnaya, M., Vinogradov, S., Gricina, I. (2015). The use of pulsed high-speed liquid jet for putting out gas blow-out. The International Journal of Multiphysics, 9 (1), 9-20. doi: https://doi.org/10.1260/1750-9548.9.1.9

33. Kustov, M. V., Kalugin, V. D., Tutunik, V. V., Tarakhno, E. V. (2019). Physicochemical principles of the technology of modified pyrotechnic compositions to reduce the chemical pollution of the atmosphere. Voprosy khimii i khimicheskoi tekhnologii, 1, 92-99. doi: https://doi.org/10.32434/0321-4095-2019-122-1-92-99

34. Vasyukov, A., Loboichenko, V., Bushtec, S. (2016). Identification of bottled natural waters by using direct conductometry. Ecology, Environment and Conservation, 22 (3), 1171-1176.

35. Pospelov, B., Rybka, E., Meleshchenko, R., Borodych, P., Gornostal, S. (2019). Development of the method for rapid detection of hazardous atmospheric pollution of cities with the help of recurrence measures. Eastern-European Journal of Enterprise Technologies, 1 (10 (97)), 29-35. doi: https://doi.org/10.15587/1729-4061.2019.155027 